\title{
Über persönlichkeitsbildende Literatur für Ärzte. Heute: das Guinness-Buch der Weltrekorde
}

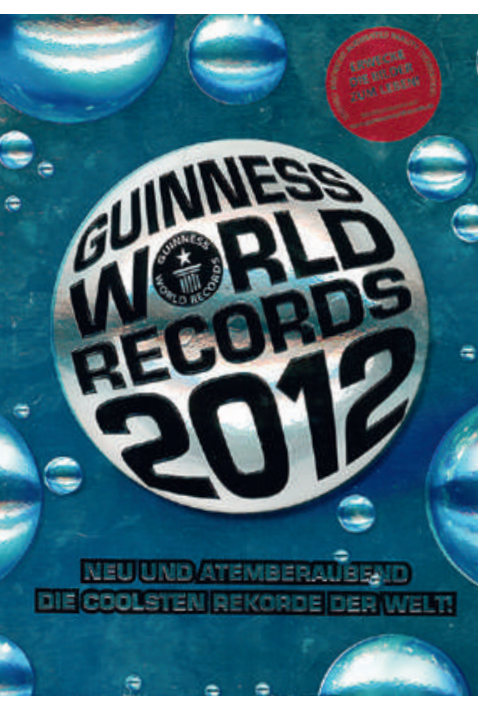

* PD Dr. rer. soc. Eberhard Wolff ist Kulturwissenschaftler, Medizinhistoriker und Mitglied der Redaktion Medizingeschichte der Schweizerischen Ärztezeitung.

\section{Literatur}

1 Bauer W. Es wird wieder Zeit für Osler: «Osler's Ten». Schweiz Ärztezeitung. 2012;93(12):474.

2 (Anonym). Guinness World Records 2012. Neu und atemberaubend die coolsten Rekorde der Welt! Mannheim: Bibliographisches Institut; 2012.

3 Simic C. Die Muse des Stillen Örtchens. Neue Zürcher Zeitung, 28. April 2012. S.49.

4 www.guinnessworldrecords. com/about/

eberhard.wolff[at]saez.ch
Im Heft zwölf warb der Redaktionskollege Bauer an dieser Stelle für den Einbezug der schöngeistigen Literatur in die Aus- und Weiterbildung der Ärzteschaft zur «Entwicklung einer Arztpersönlichkeit» [1]. Angeregt wurde er durch William Oslers mehr als hundertjähriges Bonmot, man solle sich doch als Morgenoder Bettlektüre täglich für ein halbes Stündchen etwa Plutarch, Shakespeare oder eines der Testamente zu Gemüte führen, um sich so durch inner education zum wahren Gentleman zu formen.

Wohl gesprochen! Als hie und da Verantwortlicher in Aus- und Weiterbildung der geschätzten Ärzteschaft hat es mich auch in der Schreibfeder gejuckt. «Zugeflogen» (wie der Kollege Bauer dies passend formuliert) ist mir ein «wichtiges Werk der Weltliteratur» aus der Klassikersammlung des Horts unserer Kinder, wo es schnell zum Objekt der Begierde der noch nicht so ganz persönlichkeitsgeformten Jugend avancierte: das «Guinness-Buch der Weltrekorde», hier kurz: GBWR [2]. Ich stelle anheim, es in die illustre Runde aufzunehmen. Immerhin ist das GBWR angeblich die weltweit am meisten verkaufte Buchreihe mit Copyright aller Zeiten.

Und wenn Ihnen nun Bedenken erwachsen, dabei könne es sich doch um ein Exemplar jener Schmutz- und Schundliteratur handeln, die unsere Jugend verdirbt, kann ich Sie beruhigen. Es wird in deutscher Ausgabe (einigermassen wohlfeil) vom angesehenen Bibliographischen Institut (Mannheim, Dudenstrasse!) verlegt, das sonst vor allem für eines der seriösesten Buchprodukte überhaupt bekannt ist. Und der angesehene Literat Charles Simic machte neulich in der NZZ aus seinem Herzen keine Mördergrube und bekannte, dass er das GBWR zu den Favoriten seiner Lektüre auf der Toilette zähle [3] - einem Ort, der eine unerhörte Nähe zu jenem Dressing table aufweist, von dem aus Osler seinen Plutarch zur Hand zu nehmen pflegte.

Ich wage hier zu behaupten, dass das GBWR zur Weltliteratur zählt und seine eingehende Lektüre der Persönlichkeitsbildung der jungen - und vielleicht noch mehr der in die Jahre gekommenen - Ärztinnen und Ärzte ungemein dienlich ist. Es mag uns zwar weder zu Gentlemen noch zu Ladies umschmieden, aber mit dem GBWR lässt sich ein Blick auf die Welt und den Menschen erhaschen, wie sie sich uns heute darbieten.

Das GBWR ist laut Eigeneinschätzung «a means of understanding your position in the world.» [4] Wenn das nicht persönlichkeitsbildend wirkt! An ihm kann man die Welt und sich aus der Perspektive der Ex- treme erfahren, wie etwa den kleinsten Fadenwürmern oder der grössten Kaugummiblase. Dabei dient es sogar der medizinischen Fortbildung. So erfährt man, dass der älteste praktizierende Arzt einhundert Jahre alt war, das älteste (wieder) praktizierende Bakterium aber 250 Millionen Jahre, und der schnellste Krankenfahrstuhl $111 \mathrm{~km} / \mathrm{h}$ fuhr (allerdings getunt). Und wussten Sie, wie gross die grösste Versammlung von Personen im Krankenschwesternkostüm angeblich war und wo sie stattgefunden hat? Lösung auf S. 262 des aktuellen GBWR unten. Sie werden sich wundern.

Das GBWR hält uns Menschen einen moralischen Spiegel vor. Es lässt uns mit seinen maschinengewehrartig abgefeuerten Weltrekordsalven erkennen, wie leistungsorientiert, kompetitiv und zahlenfixiert unsere Welt ist. Es offenbart, wie viele von uns von dem einen Wunsch getrieben sind, als Weltrekordler einmal «Helden für einen Tag» zu sein (vgl. D. Bowie). Sogar Schweizer zählen darunter. Und es zeigt unsere Schwäche, gerne heimlich oder offen, aber seriös legitimiert, Kuriositäten zu bestaunen wie die längste Zunge oder die grösste Niere eines Menschen. Ähnliches finden wir allerdings ab und zu auch in der ärztlichen Fachpresse.

Das GBWR ist Welt-Literatur im wahrsten Sinn des Wortes. Seine Rekorde, seine Rekordhalter wie auch seine Leser kommen aus der ganzen globalisierten Welt von Albanien bis Zimbabwe. Sie alle eint eine Passion: die Liebe zum Weltrekord.

Das GBWR ist ausserdem hochmodern, weil die Inhalte dieses Klassikers nicht wie ehedem von einem oder wenigen Experten geschaffen werden, sondern wie in der Wikipedia von einer Unzahl einzelner Beiträger. Das GBWR ist so etwas wie eine gedruckte Schwarmintelligenz à la Web 2.0.

Schliesslich spiegelt das GBWR die wachsende Multi- und Intermedialität unserer Zeit. Das Buch ist nur noch Teil eines Mediensystems zusammen mit Guinness-Fernsehserien, Websites, Sozialen Netzwerken und nicht zuletzt auch Biergläsern. Die Ausgabe von 2012 bietet sogar den Einstieg in die Augmented Reality-Technologie. Was das ist, lassen Sie sich ggf. bitte von einer Fachperson erklären, z.B. Ihren Kindern.

Mit anderen Worten: Erkenne das GBWR und erkenne damit die Welt. Und nicht zuletzt ist es ein Muss für jedes Wartezimmer, das ungeduldige Patienten zu besänftigen hat. Es könnte hier vielleicht neben der Shakespeare-Gesamtausgabe zu stehen kommen.

Eberhard Wolff* 